\title{
The Development of a Surveillance System with Different Transmission Protocols
}

\author{
Joy Iong-Zong Chen \\ Department of Electrical Engineering \\ Dayeh University \\ Changhua,Taiwan \\ E-mail: jchen@mail.dyu.edu.tw
}

\author{
Fu Chi Ye \\ Department of Electrical Engineering \\ Dayeh University \\ Changhua,Taiwan \\ E-mail:ads130539@gmail.com
}

\begin{abstract}
In this report we adopt different communication protocols to implement an uninterrupted green energy surveillance system. There are three major sub-systems includes charging sub-system, control sub-system and liquid display sub-system. On the basis of several transmission protocols, including Bluetooth, Wifi and Zigbee capability combined with wireless transmission techniques, the proposed surveillance system is designed for monitoring a green energy system remotely. The system is evaluated and measured both using the testing data report results. The proposed surveillance system can be fully extended to several different kinds of applications, such as, health care and environmental inspection. The experimental measurement results significantly show that the developed system are well established, the measured data gathered at both sides (transmitter and receiver) is also validated by shown in the display systems.
\end{abstract}

Keywords: Bluetooth; Wifi; health care; solar surveillance; liquid display; Zigbee

\section{INTRODUCTION}

Transmission protocols play an important role in the surveillance system which bases on several real world application advantages such as low cost, easy establishment, self-organizing capacity and wide deployment for experimental research or real world practical applications. The paper [1] discussed the use of Bluetooth designed with 8 channels for energy conservation, in which the sensors are implemented to sense and monitor the environmental conditions and take decision. Finally, the authors the designed system increases only by $0.00036 \mathrm{~W} / \mathrm{hr}$ at the load terminal. For tutorial point an overview of technologies used for wired and wireless sensor networks is presented in [2]. For wireless sensor network with different applications in which some features of zigbee, enOcean, wavenis, Z-wave, Wifi and Bluetooth are also discussed. It is very important to demand side energy management for a grid connected household with a locally generated photovoltaic energy. To ensure efficient household energy management, smart scheduling of electrical appliances has been presented in [3] in which the energy management of a single household that is equipped with a grid tied rooftop PV system and a set of devices acts as the consumer and producer of electrical energy. Different transmission protocols infrastructure have spread widely in various human life applications such as health care, automotive control, military command, communications and surveillance $[4,5]$. Thus, study of the issues in each transmission protocols layer has gradually become a new research trend. These research areas include power consumption networking topology, signal processing, environmental monitoring deployment, transmission Media, etc.. It is known that the greater the number of sensor nodes in the network the more precise the results provided to the BS (base station). However, in order to reduce the number of parameters for system performance, decreasing the number of sensor nodes to the optimal number is a good method. The mobility sensors is also an important point that can be applied to solve the coverage hole problem in WSNs $[6,7]$. Combining WNSs with RFID (radio frequency identification) devices is another recent study issue. For example, they can be combined together to solve surveillance issues $[8,9]$. Traditionally, wired transmission is the normal technique applied in energy monitoring. Wired communication was applied in monitoring the solar energy with project results reported in [10-11]. The system parameters in solar energy systems include the current, voltage and photovoltaic transformation power. Numerous publications have addressed these issues. Authors in [11] proposed a monitoring system design based on solar energy for a greenhouse wireless sensor network. The electrical power usage of facility electrical devices was periodically obtained from each of the wireless sensor devices in [12]. In [13] the authors present an overview of the different energy harvesting technologies and the energy saving mechanisms for WSN. The related research issues on energy efficiency for sensor networks using energy harvesting technology are then discussed. The authors' claimed that the system combined energy management with energy transfer, ensuring the energy collected by the solar energy batteries would be used reasonably. Photovoltaic systems with managed output and methods for managing output variability from photovoltaic systems are described in [14]. A plurality of the photovoltaic modules was configured to receive and convert solar energy. A photovoltaic monitoring system was designed to provide general parameters $[15,16]$, e.g., energy conservation and stability, for a solar energy monitoring system with the selected parameters used for the photovoltaic cells and controller [17, 18]. In [19] a method and system for non-invasive sensing and monitoring a processing system employed in semiconductor manufacturing was disclosed. A utility model that discloses a photovoltaic greenhouse monitoring system based on a WSN is proposed in [20]. This system was comprised of wireless information collecting nodes with high reliability and easy to expand. Recently, the IOT (internet of thing) concept has become a very important technique applied to sensing, communication and networking. 
In the near future it will be applied mostly in monitoring and surveillance systems imbedded into internet environments [21].

Green energy applications are gradually achieving an important position in industry development due to the shortage of renewable energy. Certainly, the issue of efficiency promotion in solar system to complete energy transformation becomes relatively very important. The requirement for a wireless surveillance system for "looking at" a charging system is necessary. This paper proposes a fresh idea to implement an uninterrupted solar surveillance system with a radio system. The proposed monitoring system is an ubiquitous system, i.e., it can be deployed at any location where a solar system is established. This system is designed to be initially established as a prototype model that can be commercialized after implementation and the measurements are completed. The measurement results of the implemented monitoring system provide useful results for the solar energy parameters to the operator who may not reside at the site via Internet protocol communication systems. The measurements are held at the campus around Dayeh University located in Central Taiwan (R.O.C) at a 2.2 acre site [22].

\section{SyStEM ARCHITECTURE CLAIMS}

The proposed solar energy monitoring system has three sub-systems, including the charging sub-system, the controlling sub-system and display sub-system, as illustrated in Figs. 2 (a) and (b), respectively.

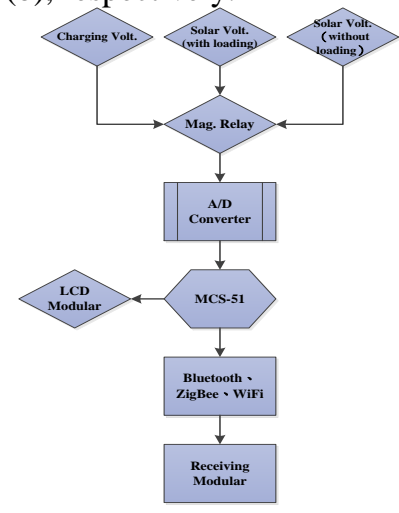

Figure 1. System Architecture

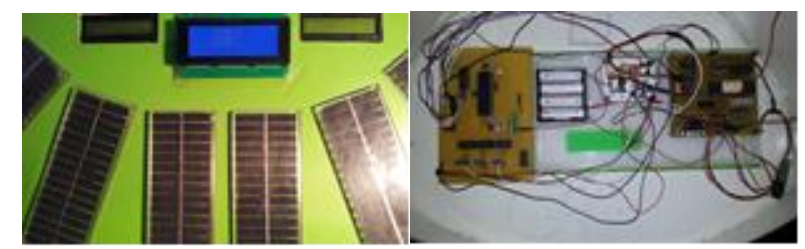

Figure 2. (a)The prototype system of green energy surveillance system (exterior) (b)The prototype system of green energy surveillance system (interior)

\section{A. Charging Sub-system}

The charging sub-system gathers energy through the solar cells, that is, the energy will be charged by the solar cell (photovoltaic) system and the energy will be stored in a long consuming battery. Back-up charging will be provided by the city power system if the energy cannot be maintained at a threshold value by the solar system. To maintain the system capable of running non-interrupted, certain signals will be captured through the system nodes utilizing RFID (radio frequency identification) techniques. The system is presented in Figs. 2(a) in which components identified, solar cell plant, also called PV (photovoltaic) array cell (or solar cell panel) with 260 Watt, a DC $12-\mathrm{V}$ charging battery, a battery with about $0.5 \mathrm{KVA}$ power in the charging subsystem. After the waveform shaper filters the energy generated from the charging sub-system, power is provided to all of the other sub-systems. By the way, the 0.5KVA power charging battery is also providing full controlling sub-system described in the following with extra power.

\section{B. Controlling Sub-system}

The controlling sub-system, as shown in Fig. 2(b), records and analyzes the data transmitted from the charging sub-system using an active embedded radio reader that reads the detected data passed from the tags. There will be tags attached in sub-system charging places where the active RFID system reader will read the effective data into the server via a serial port that communicates with a control server [23]. Thus, this sub-system is realized with hardware and software components described as below;

The A/D (analog to digital) converter samples, quantizes and codes the received data. A cost effective matching micro processor in the A/D device ADC0804, which is a serial product from Intersil Company with 8 bits precision, is chosen for the converter in this implementation. The ADC0804 specifications can be found in its data sheet [24]. There are 3 sample points designed to absorb alternatively the analog amplitude from the solar cell. The sampling rate can be adjusted by the crystal oscillator frequency of the MCU, hence, the transmission rate is adjustable. However, there still has an upper limitation rate depends on the specification of $\mathrm{A} / \mathrm{D}$ converter.

Based on the usual reasons for choosing a micro processor, e.g., multi-functional, simple designing, low cost and easy to acquire the chip packaged and numbered "AT89C51 PC24" from the @ATMEL company has become the candidate for this sub-system [25]. Moreover, the specified PC board serial number 89C51 packaged in PDIP (plastic dual inline package) form was selected as the main chip designated for the main circuit. It is known that the operating time clock of the 89C51 is generated from an internal oscillator (pin 18 and pin 19) with a frequency of $11.0592 \mathrm{MHz}$. Once the $89 \mathrm{C} 51$ baud rate is determined using a precision value generated from an embedded internal timer, the aforementioned operating frequency will have stability matched to the $9600 \mathrm{bps}$ (bit per second) operating baud rate of the RF (radio frequency) module which passes the solar cell messages. The 89C51 MODE 1, with 9600 band rate without parity check bit, 8 data bits and 
one stop bit, i.e., " $9600, \mathrm{~N}, 8,1$ " communication protocol, is operating in TH1 of internal timer 1 (timer 1 high bit). Moreover other operating modes are set with MODE 2 of 8 bit auto-loaded, SMODE $=0$ of single baud rate, hereafter, the right value of $\mathrm{TH} 1$ can be calculated as

$$
T H 1=256-\frac{2^{S M O D} \times f_{o s C}}{386 \times\left(R_{B}\right)}
$$

where SMOD denotes the internal TIMER model type of 89C51, $f_{\text {osc }}$ represents the crystal oscillator frequency, $\boldsymbol{R}_{B}$ is the baud rate in the communication port. For example, the internal timer 1, TH 1 , will become as

$$
\begin{aligned}
T H 1 & =256-\frac{2^{0} \times 11.0592 \times 10^{6}}{386 \times 9600} \\
& =253=F D H
\end{aligned}
$$

, when 9600 and model 0 timer baud rate are selected. Several important pin assignments are described as follows. The pin number P3.0 and P3.1 of 89C51 PORT3 are assigned as the communicating to and receiving function from the RF module, respectively. On the other hand, P3.0 and P3.1 are correspondingly connected to the TX and RX of the RF module and the P3.2 and P3.3 of PORT3 are two pins for accepting external interruption. The 8 pins $\mathrm{P} 0.0$ to P0.7 of PORT0 are designated connecting to an aligned 8 LEDs for error monitoring. The data bits (DB7 to DB0) coming out from the A/D device ADC0804 are arranged in contact with P1.7 to PT1.0 of 89C51 PORT 1 . There are 3 connectors P2.0、P2.1、P2.2 of PORT2 which are assigned to periodically (adjustable) absorb the sampled data from 3 reedy relays (DIA050000, $I_{d}=10 \mathrm{~mA}, \mathrm{~V}_{d}=5 \mathrm{~V}$, defined in the data sheet [24]) derived using 3 open collector inverters, respectively. The reedy relay operating time is $0.5 \mathrm{~ms}$ which is important for reacting to receive the signal from $A / D$ converter, i.e., the operation frequency is almost able to reach $800 \mathrm{~Hz}$. PORT2 $\mathrm{P} 2.3$ and $\mathrm{P} 2.4$ are for $\overline{\mathrm{RD}}$ and $\overline{W R}$ of ADC0804 to play as the signaling role when the data is read and written already, respectively. In contrast, P2.5 at PORT2 be linked to INTR of ADC0804 for informing the ready status which the $\mathrm{A} / \mathrm{D}$ convert is completed. Furthermore, the advantage of RF transmission in MCU 89C51 is useful for this design due to its variable transmission rate, from 1200 to $19200 \mathrm{~b} / \mathrm{s}$ which is helpful for the incremental the performance of data transfer. Finally, the overall specification can be checked at the Web page of [21]. The solar surveillance system prototype is designed with the detailed block diagram shown in Figs. 2(a) and (b).

\section{Liquid Displaying Sub-system}

The captured data from the Tag components will be detected by the active reader and the complete information passed to the end server via the RF modem modules. The system management display panel is designed and shown in Fig. 3. There is a communication protocol setting for adjusting the dual port parameters. The total count displays the gathered solar data records and there are 3 nodes for showing the voltage values including BV (battery voltage), SLV (solar with load voltage), and SWLV (solar without loading voltage) with the expression in hexadecimal format illustrated in Table 1 . The error code column illustrates the corresponding error status occurring during the monitoring interval. A column named "data file content" displays the date and time information involved in status events. All necessary information about this solar energy system can be monitored through the panel set up.

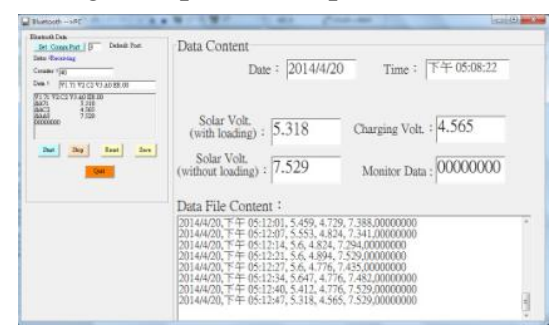

Figure 3. The gathering data from 3 different points

For evaluating the lifetime of any sensor nodes deployed in the WSN environment some conditions are taken into account in the integrated sub-systems. All consumed energy is supported by the charging sub-system.

\section{RESULTS AND SIMULATIONS}

There are 16 counts received data will be transmitted to a server established at the receiver through a RF module with band of $928 \mathrm{MHz}$. The information includes items described in the last 2.3 subsection successively shown in Fig. 3. The most important elements in the monitoring system are the stability and reliability. It is known that interference generated from the environment is the critical issue in RF transmission. The prototype system was verified using two experimental scenarios at the Dayeh university campus. The two experimental cases were set up using different RF transmission modules. The simulated solar energy system and the monitoring system were placed at the front door of Dayeh University and inside the Lab room, respectively. The distance between these two places was about 200 meters. The measured voltage data included BV, SLV, and SWLV, gathered during the afternoon hours 12:00 to 17:00 PM. The aforementioned experiment exhibited a large amount of channel fading, which included large scale fading (huge academy building, big tree), and small scale fading (pedestrians). Because the class was dismissed at the noon time 12:00 to PM 12:30 interval, the interruptions were caused mostly by people moving around the experimental area. This phenomenon was satisfactory and expected. The RF modules definitely interfered with the fading coming out of the transmission channel. It is an original idea of power management in solar cell implemented in this article. Since the real-time data of the solar cell is transmitted by RF protocol which differs from the one shown in $[5,6]$. However, the separation between the solar cell platform and the monitoring terminal will be constrained due to the RF 
protocols. Furthermore, for confirmation there are experimental results shown in Fig. 4 obtained from the simulation. The distance is about 10 meters, a pair of RF transmission modules $928 \mathrm{MHz}$ is applied. There are 3 simulated curves shown in Fig. 4 in which the results from receiving the digitalized solar cell data, therein, the curve with little triangle, the plot with little square, and the graph with little circle represent solar voltage with non-loading, solar voltage with full-loading, and the battery voltage, respectively. All the curves were collected during the period of AM 11:00 to PM 2:30 which is scaled as Y axis (time axis) and $\mathrm{X}$-axis represents the amplitude of voltage. It is reasonable to understand that the curve with little triangle is easily interrupted by shadowing, for example, the building, the pedestrians, or some tree. Besides, the other two kinds of measured voltages are much more stable. These facts significantly prove that the developed monitoring system in this article is definitely a new design.

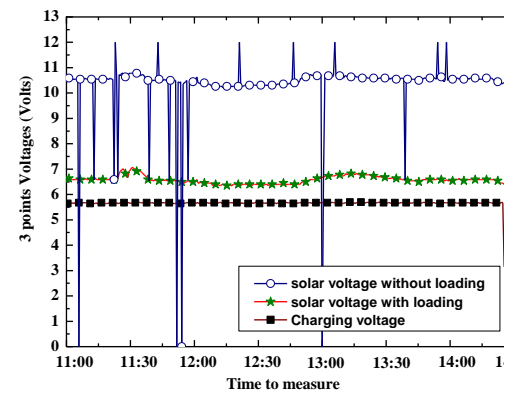

Figure 4. Measured voltage curves with indoor building to outdoor by using $928 \mathrm{MHz}$ RF transmission

\section{CURRENT \& FUTURE DEVELOPMENTS}

The RF modules, which can be alternatively replaced with Bluetooth, were adopted to transmit the necessary solar energy system information wirelessly. The WSN concept was also applied in the data gathering implementation from different places where solar energy systems could be installed. To avoid the severe shadowing effect from different transmission protocols such as ZigBee, GPRS, and Wifi, these technologies will be tested in the future in a suitable environment. The RFID techniques can be fused into the implementation for secure purposes.

\section{REFERENCES}

[1] Jaitly, S., Mahapatra, S., and Jha, A.N., "Wireless sensor based energy conservation via Bluetooth," Advance Computing Conference (IACC), 2014 IEEE International, pp. 182-185.

[2] Sharma, Hemraj and Sharma, Sukesha, "A review of sensor networks: Technologies and applications," Engineering and Computational Sciences (RAECS), 2014 Recent Advances in 6-8 March 2014, pp. 1 -4 .

[3] Adika, C.O. and Lingfeng Wang, "Autonomous Appliance Scheduling for Household Energy Management, "Smart Grid, IEEE Transactions on (Volume:5, Issue: 2 ), March 2014, pp. 673 - 682.
[4] Nobuo Tanaka, IEA, "Energy efficiency and renewable energy- A key to a better tomorrow", ISO Open Session, Cape Tow, 17 Sep. 2009.

[5] Gidlund Mikael, and Lennvall Tomas, "An Energy Efficient Method for Communication between a Wireless Sensor Network and An Industrial Control System", Patent WO 2,011,154,051A1, 2011.

[6] Zhiying Yao, and Doh Yoonmee, "Wireless sensor network and adaptive method for monitoring the security thereof", U. S. Patent $8,116,243,2012$.

[7] Weixin Wang, Jongwoo Sung and Daeyoung Kim Auto-ID Lab Korea, "Complex Event Processing in EPC Sensor Network Middleware for Both RFID and WSN", IEEE Conferences (ISORC), 2008, pp. 165-169.

[8] X, Shan, and J, Tan, "Mobile Sensor Deployment for a Dynamic Cluster-based Target Tracking Sensor Network", Intelligent Robots and Systems, 2005. (IROS 2005) 2005 IEEE/RSJ International Conference on, 2005, pp. 1452-1457.

[9] T. Kim, J. Lee, S. Lee, a. -C. Park, "Performance evaluation of RTLS based on active RFID power measurement for dense moving objects", IEICE Trans. Commun.; Vol. E92-B, No. 4, pp. 1422-1425, April 2009

[10] Wireless Sensor Network Center, http://www.wsnc.ntu.edu.tw.

[11] Bornhoevd Christof, and Mo Brian S, "Modular Monitor Service for Smart Item Monitoring", Patent U.S. 8,131,838, 2012.

[12] B. Visweswaran and Anoop R. Kulkarni, "Green Luxury"Technology and solutions for energy management", IEEE Conferences (ICM), 2009, pp. 138-140.

[13] D. Niyato, E. Hossain, and V. K. Bhargava, "Wireless sensor networks with energy harvesting technologies: A game-theoretic approach to optimal energy management", IEEE Wireless Communications, pp. 90-96, Aug. 2007.

[14] http://www.advantech.gr/ia/3175.htm, 2012.

[15] J. Hou, and Yi Gao, "Greenhouse wireless sensor network monitoring system design based on solar energy", In international conference on challenges in environmental science and computer engineering, 2010, pp. $475-479$.

[16] Pfeifer Ray, and Pandey Raju, "Apparatus and Methods for Managing Power Capacity in Data Centers Using a Wireless Sensor Network," U. S. Patent 0,054,527, Mar. 01, 2012.

[17] B. Greg, and C. Matt, "Photovoltaic System with Managed Output and Method of Managing Variability of Output from A Photovoltaic System”, U. S. Patent 0,224,831, Sep. 15, 2011.

[18] L. Bin, C. Yanbo, and Z. Lihua, "The design of photovoltaic monitoring system", In Power electronics systems and applications (PESA), 2011, pp. 1-4.

[19] K. Sanjeev, and S. Kenji, "Method of Monitoring a Semiconductor Processing System Using a Wireless Sensor Network", U. S. Patent $8,026,113$, Sep. 27, 2011.

[20] X. Zhong, and F. Wang, "Photovoltaic Greenhouse Monitoring System Based on WSN (Wireless Sensor Network)", C. N. Patent 202,041,835, Nov. 16, 2011.

[21] G. Wu, S. Talwar, K. Johnsson, N. Himayat, and K. D. Johnson, "M2M: From mobile to embedded internet", IEEE Communications Magazine, Vol. 49, No. 4, pp. 36-44, 2012.

[22] http://www.dyu.edu.tw/english/1100.php

[23] Intersil Americas Inc., “ ADC0803 、 ADC0804 8-Bit, Microprocessor-compatible, A/D converters data sheet” , FN3094, Aug 4, 2002.

[24] Atmel Corporation.8-bit Microcontroller with 4K Bytes Flash AT89C51. Available From: http://www.atmel.com/atmel/acrobat/doc0265.pdf.

[25] ICtec Company.433MHz 200 meter RF module serial no. : A060016. Available From: http://www.icci.com.tw. 